\title{
Visualization of Water-induced Surface Segregation of Polarons on Rutile $\mathrm{TiO}_{2}(110)$
}

Chi M. Yim, ${ }^{\dagger, \nabla}$ Ji Chen, ${ }^{\dagger, \nabla}$ Yu Zhang, ${ }^{\dagger}$ Bobbie-Jean Shaw, ${ }^{\dagger}$ Chi L. Pang, ${ }^{\dagger}$ David C.

Grinter,${ }^{\dagger}$ Hendrik Bluhm, ${ }^{\S} \|$ Miquel Salmeron, ${ }^{\perp}$ Christopher A. Muryn, ${ }^{\#}$ Angelos

Michaelides, ${ }^{\ddagger}$ and Geoff Thornton ${ }^{*}, \dagger$

$\dagger$ Department of Chemistry and London Centre for Nanotechnology, University College London, 20 Gordon Street, London WC1H OAJ, UK

¥Department of Physics and Astronomy, London Centre for Nanotechnology and Thomas Young Centre, University College London, London WC1E 6BT, UK

$\S$ Chemical Sciences Division, Lawrence Berkeley National Laboratory, Berkeley, California 94720, USA

" Advanced Light Source, Lawrence Berkeley National Laboratory, Berkeley, California 94720, USA

$\perp$ Materials Science Division, Lawrence Berkeley National Laboratory, Berkeley, California 94720, USA

\# School of Chemistry, The University of Manchester, Manchester, M13 9PL, UK

*Corresponding author: g.thornton@ucl.ac.uk 


\section{ABSTRACT}

Water-oxide surfaces are ubiquitous in nature and of widespread importance to phenomena like corrosion as well as contemporary industrial challenges such as energy production through water splitting. So far a reasonably robust understanding of the structure of such interfaces under certain conditions has been obtained. Considerably less is known about how overlayer water modifies the inherent reactivity of oxide surfaces. Here we address this issue experimentally for rutile $\mathrm{TiO}_{2}(110)$ using scanning tunneling microscopy and photoemission, with complementary density functional theory calculations. Through detailed studies of adsorbed water nanoclusters and continuous water overlayers, we determine that excess electrons in $\mathrm{TiO}_{2}$ are attracted to the top surface layer by water molecules. Measurements on methanol show similar behavior. Our results suggest that adsorbateinduced surface segregation of polarons could be a general phenomenon for technologically relevant oxide materials, with consequences for surface chemistry and the associated catalytic activity.

Table of Contents (TOC) Graphic

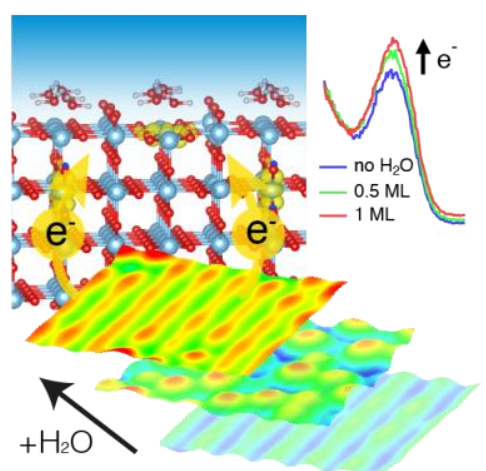


All real materials contain defects, ranging from atomic vacancies, to interstitials, to impurities, to excess electrons, and more. Defects greatly affect the physical and optical properties of materials and when present at the surfaces of materials can significantly alter the chemical activity of a material. Indeed, a large body of work has shown that defects at the surfaces of materials act as preferential sites for adsorbate binding; sites at which adsorbates are pinned or bonded more strongly and sites with altered catalytic properties. ${ }^{1-3}$ However, considerably less is known about the other side of this interplay between adsorbates and defects, namely how adsorbates alter the distribution of defects at the surfaces of materials. ${ }^{4,5}$ This is the issue we address in the current study through a detailed set of experiments and simulations of water on rutile $\mathrm{TiO}_{2}$, in which we focus on the interplay between water and excess electron defects.

The water/rutile $\mathrm{TiO}_{2}(110)$ interface has been selected for this study because of its importance to catalysis and photocatalysis and because it is one of the most widely studied and most well understood of all model oxide surface systems., , $^{3-8}$ Thanks to this earlier work we now have a broad understanding of the behavior of water on $\mathrm{TiO}_{2}(110)$ at a range of temperatures and water coverages. It is known, for example, that water dissociates at defects on $\mathrm{TiO}_{2}(110) .{ }^{9-13}$ Higher exposure to water at $<295 \mathrm{~K}$ results in molecular water adsorption at fivefold coordinated $\mathrm{Ti}\left(\mathrm{Ti}_{5 \mathrm{c}}\right)$ sites, giving rise to the first water layer, ${ }^{14-16}$ with some degree of water dissociation. ${ }^{17,18}$ Water monomers and dimers ${ }^{19}$ have been observed in scanning tunneling microscopy (STM), along with strand-like features. ${ }^{14,20}$ The latter have been ascribed to continuous hydrogen bonded water chains based on their weak corrugation in STM. ${ }^{20}$

The behavior of excess electrons on the clean $\mathrm{TiO}_{2}(110)$ surface has also been widely elucidated. ${ }^{21-27}$ However, although there has been a theoretical study of 
water/anatase interfaces, ${ }^{4}$ the impact of water on the excess electrons on rutile remains poorly understood. Here we probe the first layer of adsorbed water on rutile $\mathrm{TiO}_{2}(110)$ with STM, photoemission, and density functional theory (DFT). We find that adsorbed water molecules draw electrons from within $\mathrm{TiO}_{2}$ to the surface. This electronic coupling is also evidenced in photoemission under near ambient conditions, with a similar behavior observed for methanol.

We start by discussing our measurements of various water structures on $\mathrm{TiO}_{2}(110)$ performed in the filled states imaging mode in STM, which draw attention to the interaction of water molecules with excess electrons on the surface. More conventional empty-states images are also provided for comparison. When imaging $\mathrm{TiO}_{2}(110)$ in filled states STM (with the sample bias set at $-1 \mathrm{~V}$ ), electrons are sampled from the Fermi level $\left(E_{\mathrm{F}}\right)$ to the band-gap states (BGS) peak (located at $1 \mathrm{eV}$ below $E_{\mathrm{F}}$ ) of the $\mathrm{TiO}_{2}$ sample. As a consequence, in addition to the bright Ti-dark $\mathrm{O}$ row contrast that is usually seen in the empty- states image (inset of Figure 1a), the filled states image also exhibits lobe-like features along the $\mathrm{Ti}_{5 \mathrm{c}}$ rows (inset of Figure 1b). The lobe-like features correspond to the BGS electrons (or excess electrons) in $\mathrm{TiO}_{2}(110)$ created as a result of $\mathrm{O}_{\mathrm{b}}$-vac formation (marked by black dots in the insets of Figure 1, a and b). ${ }^{21,22,28}$ 



Figure 1. Small water clusters, nanostructured and continuous water chains on rutile $\mathrm{TiO}_{2}$ (110). (a-b) Simultaneously recorded (a) empty- and (b) filled- states (dual-mode) STM images of $r$ - $\mathrm{TiO}_{2}(110)$, recorded after exposure to $0.65 \mathrm{~L} \mathrm{H}_{2} \mathrm{O}$ at $78 \mathrm{~K}$. Circles and rectangles mark water monomers and dimers (or trimers) on the $\mathrm{Ti}_{5 \mathrm{c}}$ rows, respectively. Insets of $(\mathrm{a}-\mathrm{b})$ : Dual-mode images recorded in the vicinity of an $\mathrm{O}_{\mathrm{b}}$-vac (marked by a black dot) on $r-\mathrm{TiO}_{2}$. An arrow indicates one of the lobe-like features on the $\mathrm{Ti}_{5 \mathrm{c}}$ rows. (c) Line profiles taken along two strands in (a-b), and that taken along one section of the continuous water chain in (e-f). Solid (dashed) lines correspond to line profiles taken from the empty- (filled-) states images in $(\mathrm{a}, \mathrm{e})[(\mathrm{b}, \mathrm{f})]$ (d) Empty states STM image taken from the surface in (a) after annealing to $146 \mathrm{~K}$. The imaged area consists of both the disordered and perfect regions (see definitions in the main text). (e-f) Dual-mode STM images of the perfect region. Circles mark subsurface charge impurities. STM images in (a-b) were taken at $78 \mathrm{~K}$, those in (d-f) at $146 \mathrm{~K} .(\mathrm{g}) \mathrm{He} \mathrm{I}(h v=21.2 \mathrm{eV})$ band-gap state (BGS) region UPS spectra of the as-prepared $\mathrm{TiO}_{2}(110)$ surface (red), and that with 0.5 (green) and $1 \mathrm{ML}$ (blue) $\mathrm{H}_{2} \mathrm{O}$. All spectra were recorded at a grazing emission angle of $15^{\circ}$ from the surface [001] azimuth, at $200 \mathrm{~K}$. All the UPS spectra presented are raw data. Error bars are represented by the scatter in the data points. See Figure S10 for the corresponding He II $(h v=40.8 \mathrm{eV})$ spectra.

Exposing a $\mathrm{TiO}_{2}(110)$ surface that has been reduced to form oxygen vacancies [ $r$ - $\left.\mathrm{TiO}_{2}(110)\right]$ to $0.65 \mathrm{~L}$ Langmuirs $\left(1 \mathrm{~L}=1.33 \times 10^{-6}\right.$ mbar.s $)$ of water at $78 \mathrm{~K}$ leads to a number of water structures on the $\mathrm{Ti}_{5 c}$ rows (Figure 1a). These include water monomers (marked with circles), dimers (or trimers) (marked with rectangles), ${ }^{19}$ and 
strand-like features (or strands). ${ }^{14,20}$ Based on the very weak corrugation measured along the strands in the empty-states STM, Lee et al. ${ }^{20}$ proposed that they are continuous water chains, i.e. with water molecules adsorbed on every $\mathrm{Ti}_{5 c}$ site along the row. Instead, our STM data reveal that they are not continuous water chains: in the filled states STM (Figure 1b), each strand is further resolved into a number of protrusions, giving an overall corrugation of $\sim 80 \mathrm{pm}$ along the strands (Figure 1c). Contrary to previous work, we propose that the strands shown in Figure 1, a and b are nanostructured chains, consisting of discrete water monomers and dimers (and/or trimers) separated by at least two lattice spacings along the [001].

To substantiate the suggestion that the strands observed at $78 \mathrm{~K}$ are not continuous, we attempted to form continuous water chains by heating the surface to $146 \mathrm{~K}$ [see SI, Section S3 additional details]. The imaged area now consists of two different regions (Figure 1d). One region, enclosed with a dashed outline, consists of water monomers, dimers (or trimers) and strands that we refer to as the disordered region. Another region, enclosed with a solid outline, consists of nearly perfect rows running along the [001] direction, which we refer to as the perfect region. Line profile taken across both regions (Figure S1) indicates that both the rows in the perfect region and strands in the disordered region have the same height.

Our high-resolution STM images (Figure 1, e and f) provide evidence that the rows in the perfect region have different internal structure as compared to the strands. Unlike the strands that are further resolved into separate protrusions in the filled states STM (Figure 1b), the bright rows in the perfect region have very weak corrugation along the row direction (Figure 1f). Note that some parts of the perfect region (marked in dashed circles) exhibit significantly increased corrugation in both emptyand filled- states STM, which we attribute to $\mathrm{Ti}^{3+}$ interstitials that lie in the sub- 
surface region of $\mathrm{TiO}_{2}(110) .{ }^{29}$ On this basis, we assign the rows in the perfect region to continuous water chains. As these chains are formed on each $\mathrm{Ti}_{5 \mathrm{c}}$ row, a complete monolayer (ML) of water film is formed where $1 \mathrm{ML}$ corresponds to the number of $\mathrm{Ti}_{5 \mathrm{c}}$ sites.

Owing to their closely-packed nature, we do not expect the water molecules within a continuous water chain to exhibit any noticeable contrast along the row direction, even in the filled-states image. Nevertheless, a small corrugation is observed in the dashed line profile of $\mathrm{C}$ in Figure 1f, as well as in the rows to left of line $\mathrm{C}$. We ascribe this to coupling with electron polarons that are attracted to the surface by the water molecules. The band gap states (BGS) that are sampled in the filled-states images are polaronic in origin and hence form the contrast in the image. ${ }^{21,22,28}$

To test these ideas we performed DFT calculations on clean and watercovered surfaces containing an $\mathrm{O}_{\mathrm{b}}$-vac. In the SI, Section $\mathrm{S} 4$ we show the continuous water chain indeed has slightly preferred adsorption energy, whereas nanostructured water chain is likely to form due to kinetics. With these in mind, we now discuss the impact of water on surface polarons when continuous water chains are adsorbed on all the $\mathrm{Ti}_{5 \mathrm{c}}$ rows. Because defects are involved, for these calculations we employed a Hubbard U correction ${ }^{30}$ (specifically PBE+U) as well as the hybrid Heyd-ScuseriaErnzerhof (HSE) functional. ${ }^{31}$ The creation of an $\mathrm{O}_{\mathrm{b}}$-vac leads to two excess electrons in the slab. To establish where these electrons reside at both the clean and water covered surfaces we generated an ensemble of 20 surface models in which the $\mathrm{Ti}$ atoms in the first and second surface layer were randomly displaced from their optimal positions in the defect-free slab. These structures were subsequently geometry optimized and the location of the excess electrons examined. We find that for the 
clean surface the excess electrons mostly appear in the second layer (67\%), which agrees with previous studies. ${ }^{21,22,28,32}$ In the case of the water-covered surface, however, the excess electrons are mostly located in the topmost layer (75\%). Three typical configurations for the excess electrons at the water-covered surface are shown in Figure 2, a to c. In the 'Surf-1' configuration, one excess electron is localized in the first surface layer while the other is delocalized. In the 'Mixed-1' configuration, one electron is located in the first layer and another in the second layer. In the 'Sub-1' configuration both are in the second layer. Other configurations considered for the water film are shown in Figure S6. 


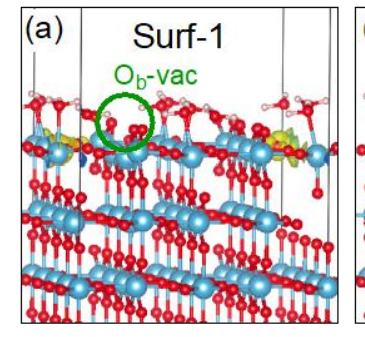

(d)

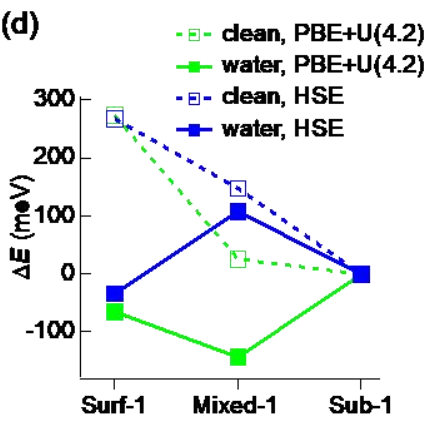



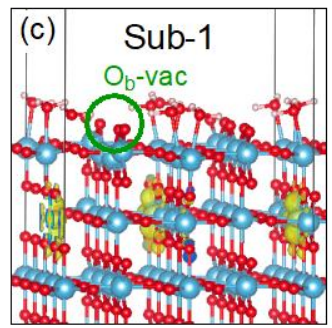

(e)

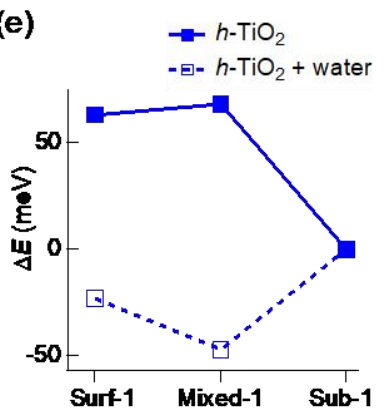

(f)

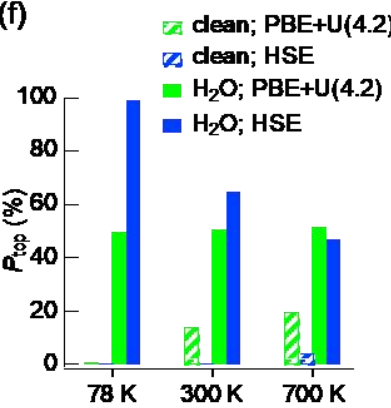

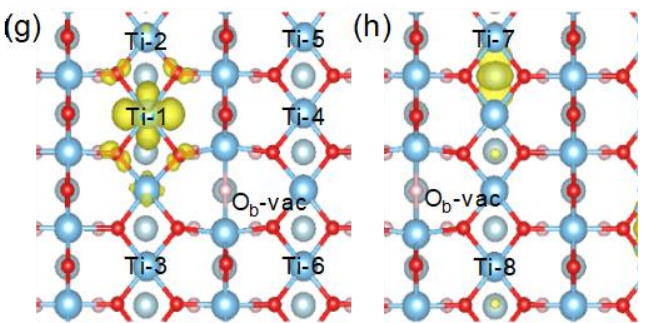

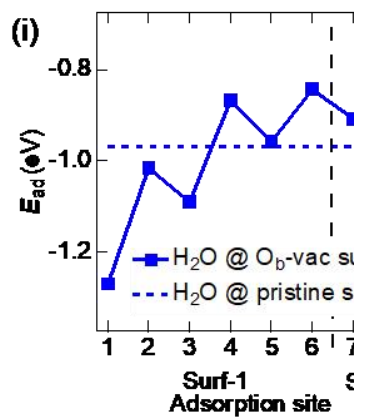

Figure 2. Stability of different excess electron configurations. (a-c) Three different locations of excess electrons induced by $\mathrm{O}_{\mathrm{b}}$-vac on the $\mathrm{TiO}_{2}(110)$ surface with $1 \mathrm{ML}$ of $\mathrm{H}_{2} \mathrm{O}$ : (a) first layer (Surf-1); (b), first and second layer (Mixed-1); (c) second layer (Sub-1). (d) Total energy difference between Surf-1, Mixed-1 and Sub-1 with and without water calculated using different methods plotted with Sub-1 as the reference. (e) Energies of Surf-1, Mixed-1 and Sub-1 configurations calculated on a hydroxylated surface model using $\mathrm{PBE}+\mathrm{U}$ ( $\mathrm{U}=4.2 \mathrm{eV}$ ). (f) Percentage of polarons in the topmost surface layer estimated according to a Boltzmann distribution, $P(E) \mu \exp (E / k T)$, where $k$ is Plank's constant, $T$ is temperature, and $E$ is the total energy of each polaron configuration. (g) Surf-1 and (h) Sub-1 configurations for excess electrons. The $\mathrm{Ti}$ sites labeled have been considered for the adsorption of single water and methanol molecules. (i) Adsorption energy $\left(E_{\mathrm{ad}}\right)$ of a single water molecule at sites of different Surf-1 and Sub-1 configurations. These results are based on a $(4 \times 2)$ supercell with 4 trilayers. A single $\mathrm{O}_{\mathrm{b}}$-vac is introduced in the supercells as indicated in (a-c).

Figure $2 \mathrm{~d}$ shows the energy difference between the 'Surf-1', 'Mixed-1' and 'Sub-1' configurations with and without water, calculated using the two different exchange-correlation functionals. The 'Surf-1' configuration is much less stable without water and becomes more stable when the water film covers the surface, indicating a strong water-polaron interaction that attracts the excess electrons towards the top surface layer. For the 'Mixed-1' structure, as it contains both first layer and second layer excess electrons, we see a strong dependence of the relative energy on 
the computational approach used (specifically the exchange-correlation functional). However, we show below that if we consider all configurations together, the results in terms of the location of the excess electron are less sensitive to the functional used (also see Figure S7).

Figure $2 \mathrm{e}$ shows the energy difference between the three configurations ('Surf-1', 'Mixed-1' and 'Sub-1') with and without water, calculated on a hydroxylated surface model [ $h-\mathrm{TiO}_{2}(110)$, Figure S8], using $\mathrm{PBE}+U$ with $U$ set at 4.2 eV. As shown in Figure 2f, without water the polaronic state 'Sub-1' is most stable. With water, polaronic states 'Surf-1' and 'Mixed-1' are more stable. We also note that without water, polaronic state 'Surf-1' of the $h-\mathrm{TiO}_{2}(110)$ model readily transforms to the 'Mixed-1' state after optimization, showing that the 'Surf-1' state is unstable without water. Hence, these calculations demonstrate that water stabilizes surface polarons also on $h-\mathrm{TiO}_{2}(110)$.

Assuming the population of sites for the excess electron follows a Boltzmann distribution, the proportion of excess electrons in the first layer as a function of temperature has been estimated. As reported in Figure 2f, this shows a large increase upon going from the clean surface to the water-covered surface. In the SI, we show that our results remain valid with the inclusion of more excess electron configurations and for a thicker $\mathrm{TiO}_{2}$ slab (Figures $\mathrm{S} 7$ and S9). Our estimate for the clean surface also agrees qualitatively with previous molecular dynamics sampling at high temperatures. $^{22,32}$ Our estimate of the polaron distribution is based on a description of the full potential energy surface with a limited number of representative configurations. Ab initio molecular dynamics simulations with advanced sampling techniques are desirable to further shed light on how water impacts the finite temperature dynamics of polarons. 
We have also calculated the interaction of a water monomer on different sites of the 'Surf-1' and 'Sub-1' configurations (Figure 2, g and h). In Figure 2i we plot these adsorption energies together with those on a defect free surface. We see that when the $\mathrm{H}_{2} \mathrm{O}$ is adsorbed on the same row as the excess charge, there is a significant attractive interaction. In particular, when the $\mathrm{H}_{2} \mathrm{O}$ molecule sits directly above the excess electron, the interaction can be as large as $0.3 \mathrm{eV}$. The $\mathrm{Ti}_{5 \mathrm{c}}-\mathrm{OH}_{2}$ bond length, however, shows a small contraction from 2.206 to $2.202 \AA$ upon adding an excess electron, suggesting that the total interaction increase is largely due to the stabilization of the polaron by water instead of the enhanced adsorption of water alone. Similar water-polaron coupling has recently been predicted for water at an anatase surface, where the results indicate that it should trigger water dissociation. ${ }^{4}$

Ultraviolet photoelectron spectroscopy (UPS) experiments provide further evidence for the enrichment of excess electrons at the surface upon water adsorption. The measurements were performed at a grazing emission angle of $15^{\circ}$ from the [001] azimuth. Under these conditions the sampling depth is only one-fourth of that in normal emission, leading to enhanced surface sensitivity.

Our UPS spectra in Figure $1 \mathrm{~g}$ evidence a clear increase of the BGS peak after dosing $1 \mathrm{ML}$ water on $\mathrm{TiO}_{2}(110)$ at $200 \mathrm{~K}$. There is a corresponding decrease in the work function by about $0.8 \mathrm{eV}$ that changes the kinetic energy of photoelectrons. This might have a minor effect on the spectral intensity, which should be eliminated by spectra normalization. An increase in the BGS peak intensity on exposure to $\mathrm{H}_{2} \mathrm{O}$ was also observed at $300 \mathrm{~K}$ in early work using a cylindrical mirror analyser, ${ }^{33}$ which has an acceptance angle of $42 \pm 6^{\circ}$. Such an increase has not been reported for previous UPS measurements involving low temperature $\mathrm{H}_{2} \mathrm{O}$ exposure. We ascribe this to the normal emission geometry used in those studies, giving rise to a lower surface 
sensitivity. ${ }^{34}$ The increase in BGS peak intensity therefore indicates that a greater quantity of excess electrons is located at the surface/subsurface regime when $\mathrm{H}_{2} \mathrm{O}$ is adsorbed.

So far we have found that water promotes the segregation of electrons to the $\mathrm{TiO}_{2}(110)$ surface under UHV conditions. To explore if similar behavior can be observed in a more practical environment of photochemistry, we performed a series of ambient pressure photoemission (APPES) measurements. Figure 3 shows APPES data recorded following exposure of hydroxylated $\mathrm{TiO}_{2}(110)\left(h-\mathrm{TiO}_{2}\right)$ to water vapor up to a partial pressure $\left(P_{\text {water }}\right)$ of 0.53 mbar. An increase up to $1.3 \times 10^{-4}$ mbar leads to an increase in the $\mathrm{OH}$ density, as indicated by the increase in the peaks corresponding to the hydroxyl $\sigma$ and $\pi$ molecular orbitals (Figure $3 b$ ). Dosing $\mathrm{H}_{2} \mathrm{O}$ at such relatively high pressures exposes the sample to a non-negligible partial pressure of $\mathrm{O}_{2}$ resulting from residual gas dissolved in the liquid water, an effect that is unavoidable based on the current technical limits for high pressure measurements. It is known from previous work that the resulting oxidation reaction leads to the formation of terminal $\mathrm{OH}$ groups, which saturates at $0.5 \mathrm{ML} .{ }^{35}$ The presence of this reaction explains both the increase in $\mathrm{OH}$ density and the decrease in the BGS peak (Figure 3c). 


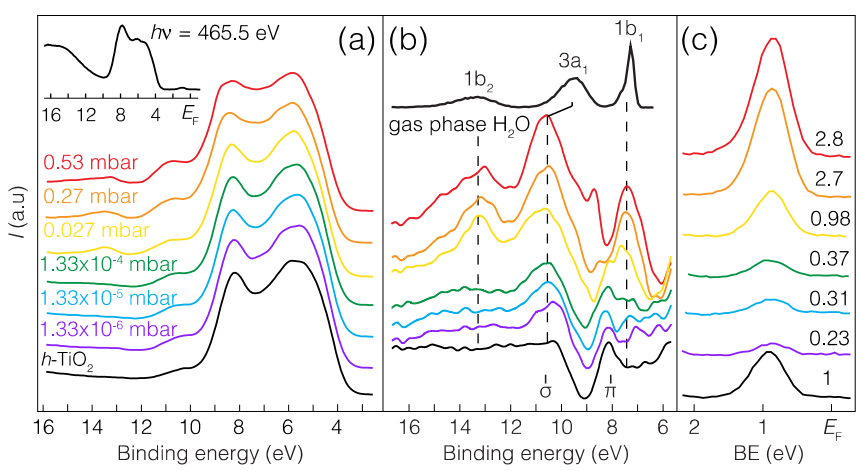

Figure 3. APPES measurements on water/ $/ \mathrm{TiO}_{2}(110)$ at increasing water vapor pressure $\left(P_{\text {water }}\right)$. (a) Valence band spectra $(h v=455 \mathrm{eV})$ acquired at incremental

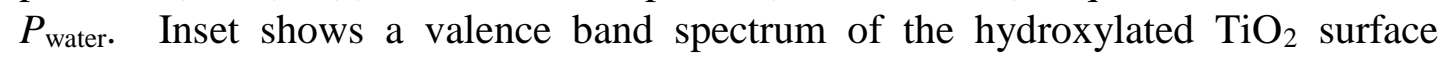
recorded at the $\mathrm{L}_{3}$ resonance energy $(h v=465.5 \mathrm{eV})(\mathrm{b})$ Corresponding difference spectra of those in (a), obtained by subtracting each spectrum from that of a clean, $\mathrm{OH}$-free surface (recorded from the as-prepared surface kept at $\sim 575 \mathrm{~K}$, not shown). In (b), the spectrum in black is of $\mathrm{H}_{2} \mathrm{O}$ in the gas-phase, obtained by retracting the sample by $2.1 \mathrm{~mm}$, aligned at the $1 \mathrm{~b}_{1}$ molecular orbital of the difference spectra. The positions of the $\sigma$ and $\pi$ hydroxyl molecular orbitals are indicated. All the spectra are vertically shifted for clarity. (c) Corresponding resonant photoemission spectra ( $h v=$ $465.5 \mathrm{eV}$ ) of the BGS peak recorded at increasing $P_{\text {water. }}$ The number above each spectrum represents the normalized intensity of the BGS peak relative to that of $h$ $\mathrm{TiO}_{2}$. All the spectra in (a-c) are presented using the same color code.

Further increasing $P_{\text {water }}$ to near ambient conditions leads to other changes in the spectra. As $P_{\text {water }}$ reaches $2.7 \times 10^{-2}$ mbar, the $\mathrm{OH}$ features do not increase further. Instead, an additional set of peaks, which correspond to $1 \mathrm{~b}_{2}, 3 \mathrm{a}_{1}$ and $1 \mathrm{~b}_{1}$ molecular orbitals of water (Figure 3b), ${ }^{14}$ start to emerge, and increase in intensity with $P_{\text {water }}$ up to its maximum value at 0.53 mbar (equivalent to a $\mathrm{H}_{2} \mathrm{O}$ coverage of $\sim 2.7 \mathrm{ML}$ ). Hydroxylation followed by molecular water adsorption was also found in an earlier APPES study. ${ }^{36}$ The corresponding increase of the BGS peak in the resonant photoemission spectra (Figure 3c) is attributed to the migration of electron polarons from deep-lying Ti interstitials attracted by water at the interface, which is reversible after removing the water (see Figure S11). This degree of enhancement is also observed off-resonance (not shown). Molecular dynamics simulations for liquid water 
on $\mathrm{TiO}_{2}(110)$ with $0.5 \mathrm{ML}$ terminal $\mathrm{OH}$ indicate that the time averaged coverage of molecular water on bare $\mathrm{Ti}_{5 \mathrm{c}}$ sites is $0.18 \mathrm{ML} .{ }^{35}$ These results indicate that the adsorbate-induced charge segregation to the surface of $\mathrm{TiO}_{2}$ also occurs in the ambient pressure range relevant to practical applications. The larger enhancement observed compared with that in Fig. 1g presumably arises because of the temperature of the substrate, with an increased mobility of electron polarons at $260 \mathrm{~K}$ compared with $200 \mathrm{~K}$.

In addition, we have performed additional STM and UPS measurements on $r$ $\mathrm{TiO}_{2}$ using methanol as a probe to show the charge-segregation on polaronic surfaces is not limited to water, but a rather general phenomenon. Our results are summarized in Figure 4. At low coverage (Figure 4a), single methanol molecules are adsorbed at the $\mathrm{Ti}_{5 \mathrm{c}}$ sites (see model in Figure $4 \mathrm{f}$ ). When two methanol molecules adsorb at adjacent $\mathrm{Ti}_{5 \mathrm{c}}$ sites, they form a $\mathrm{HB}$ with each other, becoming a dimer. ${ }^{37}$ Similar to water, methanol also appear as protrusions in the filled-states image (Figure 4b). As coverage increases, short chains composed of two dimers, as well as continuous chains of methanol molecules are also formed (Figure S13). At a saturation coverage of 0.67 ML (Figure 4c), methanol forms an ordered structure with a $L(1 \times 3)$ unit cell. ${ }^{37}$ As shown by photoemission (Figure 4d), methanol adsorption on $\mathrm{TiO}_{2}(110)$ also leads to an increase in the BGS peak intensity. This is corroborated by the results of the calculations (Figure 4e), showing that methanol, when adsorbed on $r$ - $\mathrm{TiO}_{2}$, also prefers a configuration with excess electrons in the topmost surface layer (i.e. 'Surf-1', see Figure 2). 

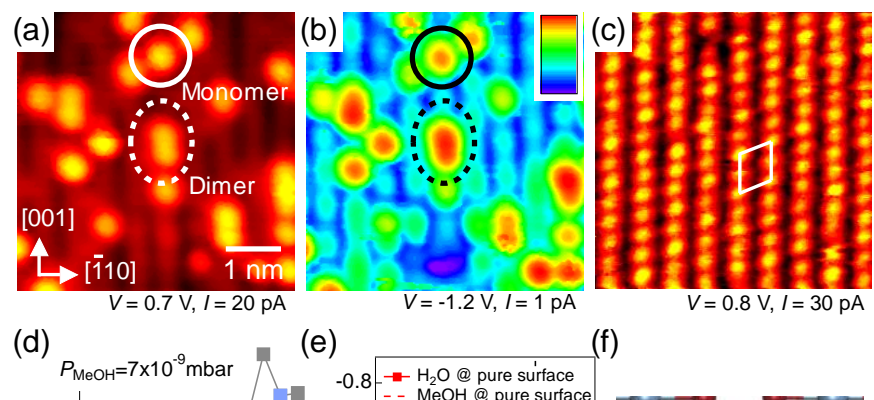

(e)

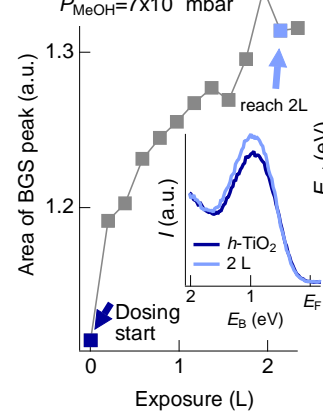

$-0.8-\mathrm{H}_{2} \mathrm{O} @$ pure surface

(f)

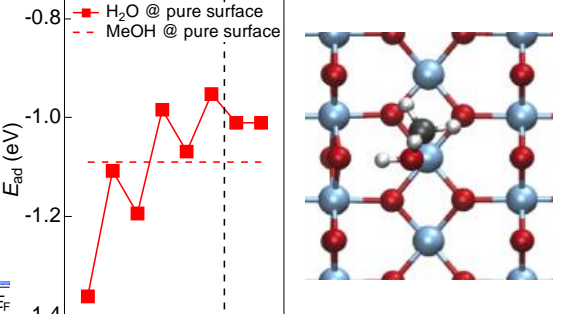

Figure 4. Methanol adsorption affects excess electron distribution in $\mathrm{TiO}_{2}$. (a-b) $6 \times 6 \mathrm{~nm}^{2}$ Simultaneously recorded (a) empty- and (b) filled- states STM images of the reduced $\mathrm{TiO}_{2}(110)$ surface recorded after a methanol exposure of $0.04 \mathrm{~L}$. Solid (dashed) lines mark methanol monomer (dimer) species. (c) $6 \times 6 \mathrm{~nm}^{2}$ empty-states image of the same surface covered with $0.67 \mathrm{ML}$ of methanol. A rhombus marks the $\mathrm{L}(1 \times 3)$ unit cell formed by methanol molecules. All STM images were recorded at $78 \mathrm{~K}$. (d) Plot of BGS peak area versus methanol exposure. The data set was obtained by collecting He I ( $h v=21.2 \mathrm{eV})$ photoemission spectra near the BGS region $\left(\sim 1 \mathrm{eV}\right.$ below $\left.\mathrm{E}_{\mathrm{F}}\right)$ while the as-prepared, $h-\mathrm{TiO}_{2}$ surface was continuously exposed to $7 \times 10^{-9}$ mbar of methanol at a sample temperature of $\sim 200 \mathrm{~K}$. The inset shows two of the spectra, taken at the start (light blue) and when methanol exposure reaches $2 \mathrm{~L}$ (dark blue), respectively. (e) Adsorption energies $\left(E_{\mathrm{ad}}\right)$ of single methanol molecules at different sites on the reduced $\mathrm{TiO}_{2}(110)$ surfaces $\left(\mathrm{O}_{\mathrm{b}}\right.$-vac) with 'Surf-1' and 'Sub-1' configurations for excess electrons (see Figure 2 for details). Dashed lines are their $E_{\mathrm{ad}}$ on the stoichiometric surface. (f) Ball and stick model of the lowest energy configuration of a single methanol molecule adsorbed at a $\mathrm{Ti}_{5 \mathrm{c}}$ site.

In conclusion, this study has shown that low temperature adsorption of water on $\mathrm{TiO}_{2}(110)$ results in discrete molecules and dimers rather than continuous chains.

Our DFT calculations indicate that continuous chains are thermodynamically stable, but kinetic barriers associated with hydrogen bond rearrangement limit their formation. Crucially, our experiments and calculations also indicate that excess electrons in $\mathrm{TiO}_{2}$ are attracted to the top surface layer by water as a result of strong- 
adsorbate-polaron coupling. A similar behavior is observed for methanol, suggesting that it is a general phenomenon. Our photoemission measurements reveal that such interactions between electron polarons in the substrate and adsorbates also occur at near ambient conditions.

The adsorbate-induced attraction of electrons to the surfaces of materials could have significant consequences for their physiochemical properties. It may cause the trapping, relaxation and recombination of photo-excited carriers, as well as band bending near the surface. Moreover, knowledge of the molecule-induced surface affinity to electron polarons may help to find specific molecule combinations leading to the control of catalytic properties of polaronic materials. Finally, enhanced segregation of electrons, beyond the levels observed here for $\mathrm{TiO}_{2}$, could even destabilize the surface itself leading to surface reconstruction or dissolution.

\section{ASSOCIATED CONTENT}

Supporting Information The Supporting Information is available free of charge on the ACS Publications website at D.O.I.:.

Experimental details, computational details, supporting sections, and Figure S1-S13 (PDF).

\section{AUTHOR INFORMATION}

Corresponding Author: *G.T.: E-mail: g.thornton@ucl.ac.uk

Author Contributions: ${ }^{\nabla}$ C.M.Y. and J.C. contributed equally to this work.

Notes: The authors declare no competing financial interest. 


\section{ACKNOWLEDGEMENTS}

C.M.Y., Y.Z., C.P. and G.T are supported by the European Research Council Advanced Grant ENERGYSURF (G.T.), European Cooperation in Science and Technology Action CM1104, and the Alexander von Humboldt Stiftung (Germany). J.C. and A.M. are supported by the European Research Council under the European Union's Seventh Framework Programme (FP/2007-2013) / ERC Grant Agreement number 616121 (HeteroIce project). We also grateful for computational resources to the UKCP consortium through EPSRC grant (EP/F036884/1), London Centre for Nanotechnology and UCL research computing. G.T. and A.M. are also supported by the Royal Society through Royal Society Wolfson Research Merit Awards. HB and MS acknowledge support by the Director, Office of Science, Office of Basic Energy Sciences, and by the Division of Chemical Sciences, Geosciences and Biosciences (HB) and Materials Sciences (MS) of the U.S. Department of Energy at LBNL under Contract No. DE-AC02-05CH11231. 


\section{References}

(1) Defects at Oxide Surfaces, Springer Series in Surface Sciences; Jupille, J., Thornton, G., Eds.; Springer International Publishing: Cham, 2015; Vol. 58.

(2) Haruta, M.; Tsubota, S.; Kobayashi, T.; Kageyama, H.; Genet, M. J.; Delmon, B. Low-Temperature Oxidation of $\mathrm{CO}$ Over Gold Supported on $\mathrm{TiO}_{2}, \alpha-$ $\mathrm{Fe}_{2} \mathrm{O}_{3}$, and $\mathrm{Co}_{3} \mathrm{O}_{4}$. J. Catal. 1993, 144, 175-192.

(3) Saavedra, J.; Doan, H. A.; Pursell, C. J.; Grabow, L. C.; Chandler, B. D. The Critical Role of Water at the Gold-Titania Interface in Catalytic CO Oxidation. Science 2014, 345, 1599-1602.

(4) Selcuk, S.; Selloni, A. Facet-Dependent Trapping and Dynamics of Excess Electrons at Anatase $\mathrm{TiO}_{2}$ Surfaces and Aqueous Interfaces. Nat. Mater. 2016, 15, 1107-1112.

(5) Cao, Y.; Yu, M.; Qi, S.; Huang, S.; Wang, T.; Xu, M.; Hu, S.; Yan, S. Scenarios of Polaron-Involved Molecular Adsorption on Reduced $\mathrm{TiO}_{2}(110)$ Surfaces. Sci. Rep. 2017, 7, 6148.

(6) Pang, C. L.; Lindsay, R.; Thornton, G. Structure of Clean and AdsorbateCovered Single-Crystal Rutile $\mathrm{TiO}_{2}$ Surfaces. Chem. Rev. 2013, 113, 38873948.

(7) Björneholm, O.; Hansen, M. H.; Hodgson, A.; Liu, L.-M.; Limmer, D. T.; Michaelides, A.; Pedevilla, P.; Rossmeisl, J.; Shen, H.; Tocci, G.; et al. Water at Interfaces. Chem. Rev. 2016, 116, 7698-7726.

(8) Mu, R.; Zhao, Z.-J.; Dohnálek, Z.; Gong, J. Structural Motifs of Water on Metal Oxide Surfaces. Chem. Soc. Rev. 2017, 46, 1785-1806.

(9) Wendt, S.; Matthiesen, J.; Schaub, R.; Vestergaard, E. K.; Lægsgaard, E.; Besenbacher, F.; Hammer, B. Formation and Splitting of Paired Hydroxyl Groups on Reduced $\mathrm{TiO}_{2}(110)$. Phys. Rev. Lett. 2006, 96, 066107.

(10) Henderson, M. A.; Epling, W. S.; Peden, C. H. F.; Perkins, C. L. Insights Into Photoexcited Electron Scavenging Processes on $\mathrm{TiO}_{2}$ Obtained From Studies of the Reaction of $\mathrm{O}_{2}$ With $\mathrm{OH}$ Groups Adsorbed at Electronic Defects on $\mathrm{TiO}_{2}(110)$. J. Phys. Chem. B 2003, 107, 534-545.

(11) Bikondoa, O.; Pang, C. L.; Ithnin, R.; Muryn, C. A.; Onishi, H.; Thornton, G. Direct Visualization of Defect-Mediated Dissociation of Water on $\mathrm{TiO}_{2}(110)$. Nat. Mater. 2006, 5, 189-192.

(12) Zhang, Z.; Bondarchuk, O.; Kay, B. D.; White, J. M.; Dohnalek, Z. Imaging Water Dissociation on $\mathrm{TiO}_{2}(110)$ : Evidence for Inequivalent Geminate $\mathrm{OH}$ Groups. J. Phys. Chem. B 2006, 110, 21840-21845.

(13) Petrik, N. G.; Kimmel, G. A. Reaction Kinetics of Water Molecules with Oxygen Vacancies on Rutile $\mathrm{TiO}_{2}(110)$. J. Phys. Chem. C 2015, 119, 2305923067.

(14) Brookes, I. M.; Muryn, C. A.; Thornton, G. Imaging Water Dissociation on $\mathrm{TiO}_{2}$ (110). Phys. Rev. Lett. 2001, 87, 266103.

(15) Liu, L.-M.; Zhang, C.; Thornton, G.; Michaelides, A. Structure and Dynamics of Liquid Water on Rutile $\mathrm{TiO}_{2}(110)$. Phys. Rev. B 2010, 82, 161415.

(16) Kimmel, G. A.; Baer, M.; Petrik, N. G.; VandeVondele, J.; Rousseau, R.; Mundy, C. J. Polarization- and Azimuth-Resolved Infrared Spectroscopy of Water on $\mathrm{TiO}_{2}(110)$ : Anisotropy and the Hydrogen-Bonding Network. $J$. Phys. Chem. Lett. 2012, 3, 778-784. Allegretti, F.; O'Brien, S.; Polcik, M.; Sayago, D. I.; Woodruff, D. P. 
Adsorption Bond Length for $\mathrm{H}_{2} \mathrm{O}$ on $\mathrm{TiO}_{2}(110)$ : A Key Parameter for Theoretical Understanding. Phys. Rev. Lett. 2005, 95, 226104.

Amft, M.; Walle, L. E.; Ragazzon, D.; Borg, A.; Uvdal, P.; Skorodumova, N. V.; Sandell, A. A Molecular Mechanism for the Water-Hydroxyl Balance During Wetting of $\mathrm{TiO}_{2}$. J. Phys. Chem. C 2013, 117, 17078-17083.

(19) Matthiesen, J.; Hansen, J. Ø.; Wendt, S.; Lira, E.; Schaub, R.; Lægsgaard, E.; Besenbacher, F.; Hammer, B. Formation and Diffusion of Water Dimers on Rutile $\mathrm{TiO}_{2}$ (110). Phys. Rev. Lett. 2009, 102, 226101.

(20) Lee, J.; Sorescu, D. C.; Deng, X.; Jordan, K. D. Water Chain Formation on $\mathrm{TiO}_{2}(110)$. J. Phys. Chem. Lett. 2013, 4, 53-57.

(21) Yim, C. M.; Watkins, M. B.; Wolf, M. J.; Pang, C. L.; Hermansson, K.; Thornton, G. Engineering Polarons at a Metal Oxide Surface. Phys. Rev. Lett. 2016, 117, 116402.

(22) Setvin, M.; Franchini, C.; Hao, X.; Schmid, M.; Janotti, A.; Kaltak, M.; Van de Walle, C. G.; Kresse, G.; Diebold, U. Direct View at Excess Electrons in $\mathrm{TiO}_{2}$ Rutile and Anatase. Phys. Rev. Lett. 2014, 113, 086402.

(23) Sezen, H.; Buchholz, M.; Nefedov, A.; Natzeck, C.; Heissler, S.; Di Valentin, C.; Wöll, C. Probing Electrons in $\mathrm{TiO}_{2}$ Polaronic Trap States by IR-

Absorption: Evidence for the Existence of Hydrogenic States. Sci. Rep. 2014, 4, 53 .

(24) Moser, S.; Moreschini, L.; Jaćimović, J.; Barišić, O. S.; Berger, H.; Magrez, A.; Chang, Y. J.; Kim, K. S.; Bostwick, A.; Rotenberg, E.; et al. Tunable Polaronic Conduction in Anatase $\mathrm{TiO}_{2}$. Phys. Rev. Lett. 2013, 110, 196403.

(25) Deskins, N. A.; Rousseau, R.; Dupuis, M. Defining the Role of Excess Electrons in the Surface Chemistry of $\mathrm{TiO}_{2}$. J. Phys. Chem. C 2010, 114, 5891-5897.

(26) Deskins, N. A.; Rousseau, R.; Dupuis, M. Distribution of $\mathrm{Ti}^{3+}$ Surface Sites in Reduced $\mathrm{TiO}_{2}$. J. Phys. Chem. C 2011, 115, 7562-7572.

(27) Deskins, N. A.; Rousseau, R.; Dupuis, M. Correction to "Localized Electronic States From Surface Hydroxyls and Polarons in $\mathrm{TiO}_{2}(110)$," "Defining the Role of Excess Electrons in the Surface Chemistry of $\mathrm{TiO}_{2}$," And "Distribution of $\mathrm{Ti}^{3+}$ Surface Sites in Reduced $\mathrm{TiO}_{2}$." J. Phys. Chem. C 2014, 118, 13326-13327.

(28) Papageorgiou, A. C.; Beglitis, N. S.; Pang, C. L.; Teobaldi, G.; Cabailh, G.; Chen, Q.; Fisher, A. J.; Hofer, W. A.; Thornton, G. Electron Traps and Their Effect on the Surface Chemistry of $\mathrm{TiO}_{2}(110)$. Proc. Natl. Acad. Sci. U.S.A. 2010, 107, 2391-2396.

(29) Yoon, Y.; Du, Y.; Garcia, J. C.; Zhu, Z.; Wang, Z.-T.; Petrik, N. G.; Kimmel, G. A.; Dohnálek, Z.; Henderson, M. A.; Rousseau, R.; et al. Anticorrelation Between Surface and Subsurface Point Defects and the Impact on the Redox Chemistry of $\mathrm{TiO}_{2}(110)$. ChemPhysChem 2014, 16, 313-321.

(30) Dudarev, S. L.; Botton, G. A.; Savrasov, S. Y.; Humphreys, C. J.; Sutton, A. P. Electron-Energy-Loss Spectra and the Structural Stability of Nickel Oxide: an LSDA+U Study. Phys. Rev. B 1998, 57, 1505-1509.

(31) Heyd, J.; Scuseria, G. E.; Ernzerhof, M. Erratum: "Hybrid Functionals Based on a Screened Coulomb Potential" [J. Chem. Phys.118, 8207 (2003)]. J Chem Phys 2006, 124, 219906.

(32) Kowalski, P. M.; Camellone, M. F.; Nair, N. N.; Meyer, B.; Marx, D. Charge Localization Dynamics Induced by Oxygen Vacancies on the $\mathrm{TiO}_{2}(110)$ 
Surface. Phys. Rev. Lett. 2010, 105, 146405.

(33) Kurtz, R. L.; Stock-Bauer, R.; Madey, T. E.; Román, E.; De Segovia, J. L. Synchrotron Radiation Studies of $\mathrm{H}_{2} \mathrm{O}$ Adsorption on $\mathrm{TiO}_{2}(110)$. Surf. Sci. 1989, 218, 178-200.

(34) Payne, D. T.; Zhang, Y.; Pang, C. L.; Fielding, H. H.; Thornton, G. Coverage-Dependent Two-Photon Photoexcitation at the $\mathrm{H}_{2} \mathrm{O} / \mathrm{TiO}_{2}$ Interface. Surf. Sci. 2016, 652, 189-194.

(35) Hussain, H.; Tocci, G.; Woolcot, T.; Torrelles, X.; Pang, C. L.; Humphrey, D. S.; Yim, C. M.; Grinter, D. C.; Cabailh, G.; Bikondoa, O.; et al. Structure of a Model $\mathrm{TiO}_{2}$ Photocatalytic Interface. Nat. Mater. 2017, 16, 461-466.

(36) Ketteler, G.; Ogletree, D. F.; Bluhm, H.; Liu, H.; Hebenstreit, E. L. D.; Salmeron, M. In Situ Spectroscopic Study of the Oxidation and Reduction of Pd(111). J. Am. Chem. Soc. 2005, 127, 18269-18273.

(37) Silber, D.; Kowalski, P. M.; Traeger, F.; Buchholz, M.; Bebensee, F.; Meyer, B.; Wöll, C. Adsorbate-Induced Lifting of Substrate Relaxation Is a General Mechanism Governing Titania Surface Chemistry. Nat. Commun. 2016, 7 , 12888. 